\title{
A Literature Survey on Enhancement of Low-Quality Fingerprint Images
}

\author{
Neethu.B PG Scholar ${ }^{1}$, Shimna.P.K ${ }^{2}$ \\ ${ }^{1}$ Department of Electronics \& Communication Engineering Vimal Jyothi Engineering College Chemberi,India \\ Assistant.Professor ${ }^{2}$ Department of Electronics \&Communication Engineering Vimal Jyothi Engineering \\ College \\ Chemberi,India
}

\begin{abstract}
Fingerprint authentication for content protection in the human-machine systems, cybernetics, and computational intelligence is very popular. Because of the complex input contexts, low-quality input fingerprint images always exist with cracks and scars, dry skin, or poor ridges and valley contrast ridges. In this paper three different methods are introduced to enhance the low quality fingerprint images.

Index Terms: Fingerprint enhancement, Unsharp masking, Quadratic filter, Fingerprint matching; Minutiae matching, Feature vector distance two-stage filtering
\end{abstract}

\section{Introduction}

Biometrics, is described as the science of recognizing an individual based on his or her physical or behavioral traits, is beginning to gain acceptance as a legitimate method for the determination of an individual's identity [1]. Biometric systems have now been deployed in various commercial, civilian, and forensic applications as a means of establishing identity in the human-machine systems, cybernetics, and computational intelligence [2], [3].

Among all the biometric indicators, fingerprints are one of the highest levels of reliability and have been extensively used by forensic experts in criminal investigations. Fingerprint is the impression left on a surface by the friction caused by the ridges on a finger or any part of hand and is a unique biometric identifier .Fingerprint recognition has emerged as one of the most reliable means of biometric authentication because of its universality, distinctiveness, permanence, and accuracy. However, the performance of fingerprint recognition techniques relies heavily on the quality of the input fingerprint images. Fingerprint images are frequently of low quality, because of the contexts of the image-acquisition process. Normally, noise from input devices can be easily eliminated using simple filters; however, the imperfection of ridge structures from each individual are not typically well defined, and it is very hard to enhance the contexts of these images. The quality of a fingerprint image may be poor or significantly different because of various factors, such as wetness and dryness, pressure strength, smears, and so on, which lead to different types of degradation in fingerprint images. For example, some ridges are not connected even if they should be, and some parallel ridges are not well separated. Thus, fingerprint-enhancement approaches are needed to deal with all of these factors.

Fingerprint recognition algorithms are roughly classified into two classes : minutiae-based and imagebased methods. A majority of the existing enhancement techniques are based on the use of spatial-domain methods, such as contextual filters or Gabor filters, anisotropic filters , compensation filters , image-scale-based filters, directional filters, and residual orientation modeling. The spatial-domain techniques involve spatial convolution of the image with filter masks, which is simple for operation. Other enhancement methods are based on using frequency-domain methods, such as Log-Gabor filters, directional Fourier filters, wavelets ,discrete cosine transform, fast Fourier transform (FFT),short-time Fourier transform (STFT)], and so on. as follows.

In this paper three different methods are introduced to enhance the low quality finger print images and

\section{Unsharp Masking Using Quadratic Filter}

This method summarizes the design and implementation of a quadratic edge detection filter based on Volterra series. The filter is employed in an unsharp masking scheme for enhancing fingerprints in a dark and noisy background. The proposed filter can account for much of the polynomial nonlinearities inherent in the input image and can replace the conventional edge detectors like Laplacian, LOG,etc. The application of the new filter is in forensic investigation where enhancement and identification of latent fingerprints are key issues.

The theory of Volterra functionals was developed by Vito Volterra to model nonlinear systems as parallel combinations of linear and polynomial systems of increasing order. Many classes of quadratic filters were developed for edge preserving noise smoothing, edge extraction, image interpolation, etc. An Nth order Volterra filter $[20,21]$ with input vector $X[n]$ and output vector $Y[n]$ is realized by 


$$
\begin{gathered}
y[n]=h_{0}+\sum_{r=1}^{\infty} \sum_{n_{1}=1}^{N} \sum_{n_{2}=1}^{N} \ldots \sum_{n_{r}=1}^{N} h_{r}\left[n_{1}, n_{2}, \ldots, n_{r}\right] . \\
x\left[n-n_{1}\right] x\left[n-n_{2}\right] \cdots x\left[n-n_{r}\right]
\end{gathered}
$$

$\mathrm{h} 0$ is the output offset when no input is present and $\mathrm{r}$ indicates the order of non linearity. The impulse response term $h_{r}\left[n 1, n 2 \ldots . . n_{r}\right]$ is $r$ th order volterra kernel

$$
\begin{aligned}
y[n] & =h_{0}+\sum_{n_{1}=1}^{N} h_{1}\left[n_{1}\right] x\left[n-n_{1}\right] \\
& +\sum_{n_{1}=1}^{N} \sum_{n_{2}=1}^{N} h_{2}\left[n_{1}, n_{2}\right] x\left[n-n_{1}\right] x\left[n-n_{2}\right]
\end{aligned}
$$

or equivalently by the matrix equation:

$Y[n]=h_{0}+X^{T}[n] H_{1}+X^{T}[n] H_{2} X[n]$

where

$X[n]=\left[\begin{array}{llll}x(n) & x(n-1) & \cdots & x(n-N+1)\end{array}\right]^{T}$

$$
H_{1}=\left[h_{1}(0) h 1(1) \cdots h_{1}(N-1)\right]^{T}
$$

and

$$
H_{2}=\left[\begin{array}{cccc}
h_{2}(0,0) & h_{2}(0,1) & \ldots & h_{2}(0, N-1) \\
h_{2}(1,0) & h_{2}(1,1) & \ldots & h_{2}(1, N-1) \\
h_{2}(2,0) & h_{2}(2,1) & \ldots & h_{2}(2, N-1) \\
\vdots & \vdots & \ddots & \vdots \\
h_{2}(N-1,0) & h_{2}(N-1,1) & \cdots & h_{2}(N-1, N-1)
\end{array}\right]
$$

\section{A.2Dquadratic filter}

The 2 dimensional qudratic filter is governed by the equation

$$
\begin{aligned}
& y\left[n_{1}, n_{2}\right]=\sum_{m_{11}=0}^{N_{1}-1} \sum_{m_{12}=0}^{N_{2}-1} \sum_{m_{21}=0}^{N_{1}-1} \sum_{m_{22}=0}^{N_{2}-1} h_{1}\left[m_{11}, m_{12}, m_{21}, m_{22}\right] \\
& \quad \times x\left[n_{1}-m_{11}, n_{2}-m_{12}\right] x\left[n_{1}-m_{21}, n_{2}-m_{22}\right]
\end{aligned}
$$

Eq. (7) can be represented in the matrix form as

$$
y\left[n_{1}, n_{2}\right]=\mathbf{X}^{T}\left[n_{1}, n_{2}\right] \mathbf{H}_{2} \mathbf{X}\left[n_{1}, n_{2}\right]
$$

The quadratic kernel $\mathrm{H} 2$ has N1N2 * N1N2 elements and each element consists of N2 2 sub-matrices H(i; j) with $\mathrm{N} 1 * \mathrm{~N} 2$ elements given as;

$$
\mathbf{H}_{2}=\left[\begin{array}{cccc}
\mathbf{H}(0,0) & \mathbf{H}(0,1) & \cdots & \mathbf{H}\left(0, N_{2}-1\right) \\
\mathbf{H}(1,0) & \mathbf{H}(1,1) & \cdots & \mathbf{H}\left(1, N_{2}-1\right) \\
\vdots & \vdots & \ddots & \vdots \\
\mathbf{H}\left(N_{2}-1,0\right) & \mathbf{H}\left(N_{2}-1,1\right) & \cdots & \mathbf{H}\left(N_{2}-1, N_{2}-1\right)
\end{array}\right]
$$

where each sub-matrix $\mathbf{H}(i, j)$ is given by

$$
\mathbf{H}(i, j)=\left[\begin{array}{ccc}
h(0, i, 0, j) & \cdots & h\left(0, i, N_{1}-1, j\right) \\
h(1, i, 0, j) & \cdots & h\left(1, i, N_{1}-1, j\right) \\
\vdots & \vdots & \vdots \\
h\left(N_{1}-1, i, 0, j\right) & \cdots & h\left(N_{1}-1, i, N_{1}-1, j\right)
\end{array}\right]
$$

The scheme proposed for enhancing fingerprint in noisy back ground. It relies on a quadratic edge detection filter. The flow of work is as depicted in Fig. 1. The first phase of work is in designing the edge detection kernel $\mathrm{H} 2$. The design is based on the minimization of mean square error between a synthetic true edge and its noisy version. The second phase is the computationally efficient implementation of $\mathrm{H} 2$ based on singular value decomposition. In the last phase, testing of the filter is done with standard images corrupted by impulsive and Gaussian noise of different noise variances and the model is validated in terms of edge preservation in the presence of noise.

\section{A. Unsharp masking with quadratic filter}

It is observed that quadratic edge detectors are very noise immune and have better edge detection characteristics than Laplacian and LoG filters. The unsharp masking scheme with quadratic edge detection filter 
is shown in Fig. 2. The edges in the input image $x[n 1, n 2]$ are separated by the quadratic filter. They are then scaled by a factor $\Lambda$ and are added with the input image to yield the enhanced version $x^{`}[n 1, n 2]$.The scale factor $\Lambda$ is chosen in such a way that there is improvement in $x^{`}[\mathrm{n} 1, \mathrm{n} 2]$ in respect of visual quality as well as in performance criteria like SNR, PSNR, etc.

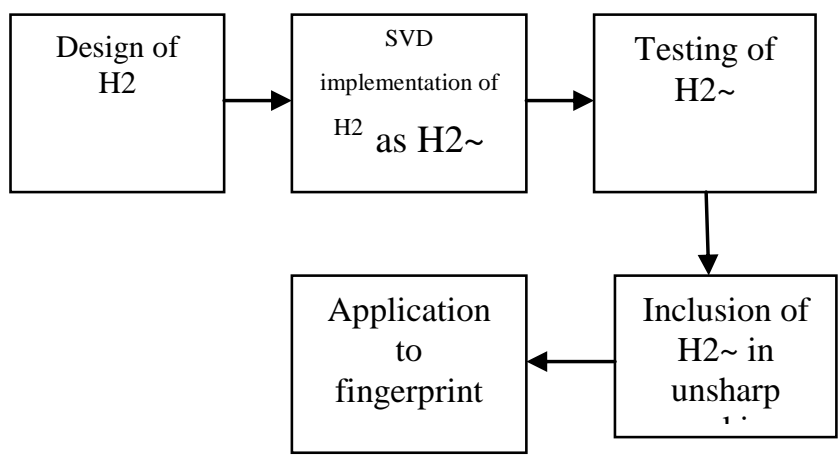

Fig1.flow of work

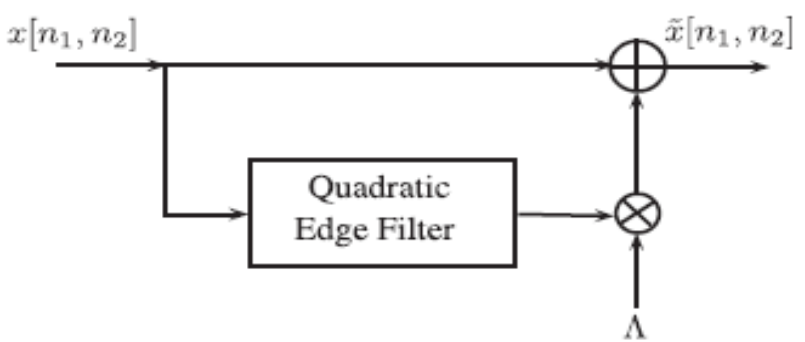

Fig. 2 Unsharp masking with quadratic filter.

III. Two-Stage Enhancement Scheme

Two-stage enhancement scheme in both the spatial domain and the frequency domain by learning from the underlying images. To remedy the ridge areas and enhance the contrast of the local ridges, first enhance the fingerprint image in the spatial domain with a spatial ridge-compensation filter by learning from the images. With the help of the first step, the second stage filter, i.e., a frequency band pass filter that is separable in the radial and angular frequency domains is employed

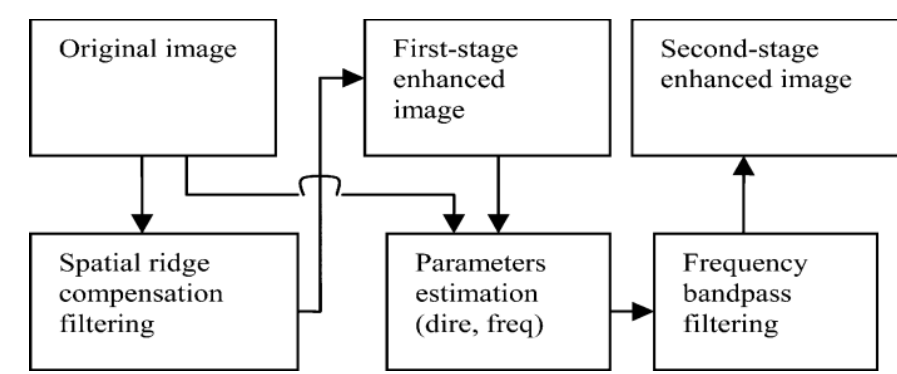

Fig. 1. Diagram of the two-stage enhancement scheme (dire $=$ ridge direction, freq $=$ ridge frequency).

\section{A.First-Stage Enhancement: Spatial Ridge-Compensation Filter}

The first stage performs ridge compensation along the ridges in the spatial field. This step enhances the fingerprint's local ridges using the neighbor pixels in a small window with a weighted mask along the orientation of the local ridges. Each pixel in the fingerprint is replaced with its weighted neighbor Sampling pixels in a small window and with the controlled contrast parameters along the orientation of the local ridges.

The main idea of the first-stage enhancement scheme is to estimate unbiased local orientation and compensate the possible defects by using the local orientation. The scheme consists of three steps: local normalization, local orientation estimation, and local ridge-compensation filtering, which are summarized as follows. 


\section{1.)Local Normalization}

This step is used to reduce the local variations and standardize the intensity distribution in order to consistently estimate the local orientation. we propose using a local normalization to reduce local variations in graylevel values. For each pixel $(i, j)$ in a subimage, which is acquired by dividing the fingerprint image into subimages first, the normalized image is defined as follows:

$$
\begin{aligned}
\operatorname{norimg}(i, j) & =M_{0}+\operatorname{coeff} *(\operatorname{img}(i, j)-M) \\
\operatorname{coeff} & =\frac{V_{0}}{V} .
\end{aligned}
$$

Here, img $(i, j)$ is the gray-level value of the fingerprint image in pixel $(i, j)$, norimg $(i, j)$ is the normalizing value in pixel $(i, j)$, and coeff is the amplificatory multiple of the normalized image. $M$ is the mean of the subimage, and $V$ is the variance of the subimage. $M_{0}$ and $V 0$ are the desired mean and variance values, respectively

\section{2) Local Orientation Estimation}

This step determines the dominant direction of the ridges in different parts of the fingerprint image. This is a critical processing, and errors occurring at this stage are propagated to the frequency filter. We used the gradient method for orientation estimation and an orientation smoothing method with a Gaussian window to correct the estimation [ 12].For number of non overlapping blocks with the size of $W \times W$, a single orientation is assigned corresponding to dominant orientation of the block. For each pixel in a block, a simple gradient operator, such as the Sobel mask [36], is applied to obtain the horizontal gradient value $G x(u, v)$ and vertical gradient value $G y(u, v)$. The block horizontal and vertical gradients, i.e., $G x x$ and $G x y$, are obtained by adding up all the pixel gradients of the corresponding direction, as in (3) and (4). Then, the block orientation $O(x, y)$ is determined using the block horizontal and vertical gradients, as

$$
\begin{aligned}
G_{x y} & =\sum_{u=i-(w / 2)}^{i+(w / 2)} \sum_{v=j-(w / 2)}^{j+(w / 2)} 2 G_{x}(u, v) G_{y}(u, v) \\
G_{x x} & =\sum_{u=i-(w / 2)}^{i+(w / 2)} \sum_{v=j-(w / 2)}^{j+(w / 2)}\left(G_{x}^{2}(u, v)-G_{y}^{2}(u, v)\right) \\
O(x, y) & =\frac{1}{2} \tan ^{-1}\left(\frac{G_{x y}}{G_{x x}}\right) .
\end{aligned}
$$

\section{3) Local Ridge-Compensation Filter}

With the estimated orientation values in place, the final step compensates the ridge artifacts using a local ridge-compensation filter with a rotated rectangular window to match the local orientation. For each pixel $(i, j)$ in the normalized image, the computing formula for the ridge-compensation filter is defined as follows:

$$
\begin{aligned}
\text { fltimg }(i, j) & =\frac{\left(\sum_{m=-(w-1) / 2}^{(w-1) / 2} \sum_{n=-(h-1) / 2}^{(h-1) / 2} \operatorname{norimg}\left(i^{\prime}, j^{\prime}\right)\right)}{(((w-1) \times \beta+\alpha) \times h)} \\
i^{\prime} & =i+m \cos (O(i, j))+n \sin (O(i, j)) \\
j^{\prime} & =j-m \sin (O(i, j))+n \cos (O(i, j))
\end{aligned}
$$

where fltimg $(i, j)$ denotes the ridge-compensation filtered image, $\operatorname{norimg}(i, j)$ is the local normalized image, $O(i$, $j$ ) is the local ridge orientation image, and $\left(i_{-}, j_{-}\right)$is the pixel coordinate in the affine transform to match the local ridge orientation. $w$ and $h$ are the width and height of the enhanced windows, respectively. $m$ and $n$ are the integer numbers and determined by $w$ and $h$.

\section{A. Second-Stage Enhancement: Frequency Bandpass Filter}

A second-stage enhancement with a tuned bandpass filter is proposed to enhance the fingerprint image serially. these methods have demerits, such as difficult parameters selection and non effective filtering. To 
overcome these shortcomings, we propose an improved scheme with an exponential bandpass filter that is explained as follows.

Using polar coordinates $(\rho, \varphi)$ to express the filters as a separable function, the frequency band pass filters $H(\rho$, $\varphi)$ used are separable in the radial and the angular domains, respectively and are given as follows:

$$
\begin{aligned}
H(\rho, \phi) & =H_{\rho}(\rho) H_{\phi}(\phi) \\
H_{\rho}(\rho) & =\frac{1}{\sqrt{2 \pi} \rho_{\mathrm{BW}}} \exp \left(-\frac{\left(\rho-\rho_{c}\right)^{2}}{2 \rho_{\mathrm{BW}}}\right) \\
H_{\phi}(\phi) & = \begin{cases}\cos ^{2} \frac{\pi}{2} \frac{\left(\phi-\phi_{c}\right)}{\phi_{\mathrm{BW}}}, & \text { if }|\phi|<\phi_{\mathrm{BW}}, \\
0, & \text { otherwise. }\end{cases}
\end{aligned}
$$

The radial filter $H \rho(\rho)$ is an exponential band pass filter with the center frequency that is defined by $\rho_{c}$ and bandwidth $\rho$ BW,

$$
\rho_{\mathrm{BW}}=\frac{C \times \rho_{c}-C}{\rho_{c}} ; \quad C \text { is a constant value. }
$$

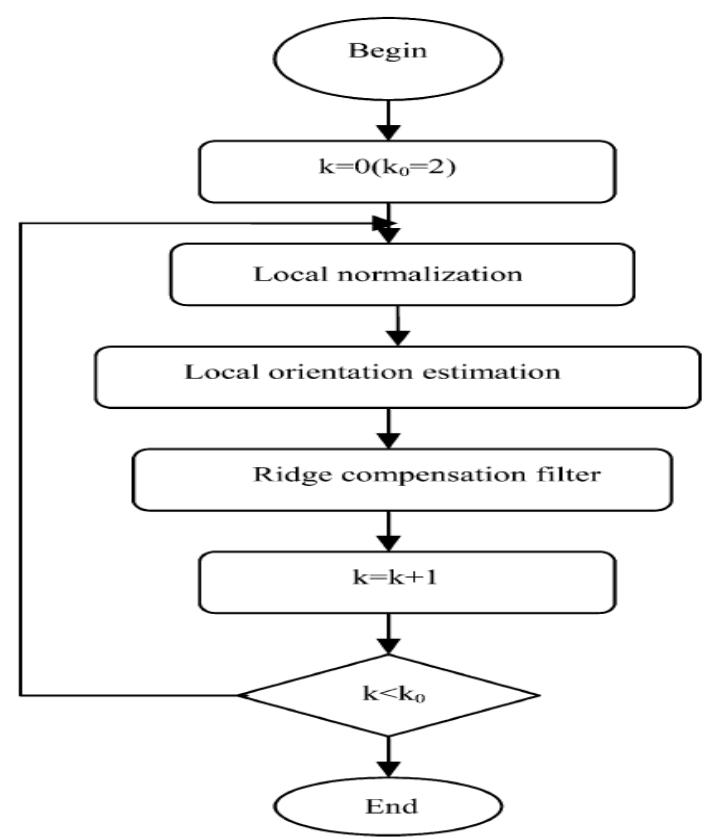

g. 3. Diagram of the whole process of the first-stage enhancement.

\section{A Medium Resolution Fingerprint Matching System}

In this technique, a novel minutiae based fingerprint matching system is proposed. The system is suitable for medium resolution fingerprint images obtained by low cost commercial sensors.

In this technique a new thinning algorithm, a new features extraction and representation, and a novel feature distance matching algorithm are presented. The proposed system is rotation and translation invariant and is suitable for complete or partial fingerprint matching. The proposed algorithms are optimized to be executed on low resource environments both in CPU power and memory space .

So he proposed fingerprint matching system consists of 5 steps,

1. Thinning of the fingerprint image.

2. Core point detection.

3. Minutiae extraction.

4. Feature vector construction.

5. Distance based matching. 


\section{A.Thinning algorithm}

For type1 ridges, the block is scanned column by column, during this scan, the local minimum is searched for in each column, and in a new block initialized by zeros, all local minima in those columns will be replaced with ones. For type 2 ridges, the block is scanned row by row, during this scan, the local minimum is searched for in each row, and in a new block initialized by zeros, and all local minima in those rows will be replaced with ones. The fingerprint image is divided into blocks of $8 * 8$ pixels and each block is processed one at a time

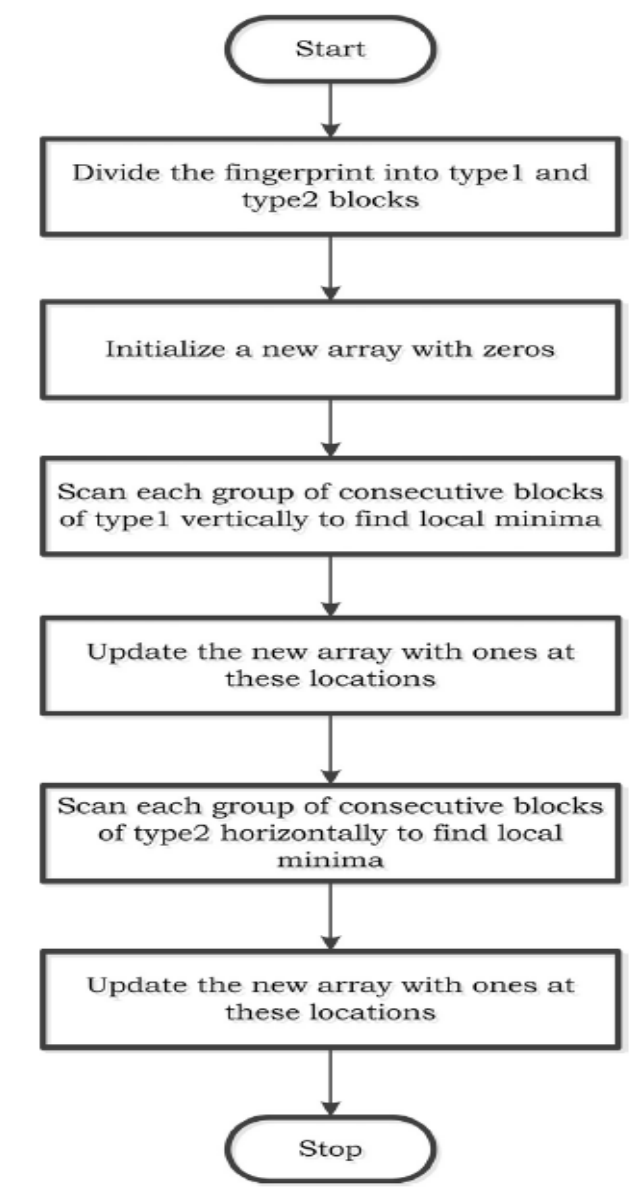

Figure 4 A flowchart of the proposed algorithm.

\section{B) Core (singular) point detection}

The core (or singular) point of a fingerprint is defined as "the

point of the maximum curvature on the convex ridge [24], which is usually located in the central area of fingerprint. The method is used to determine the core point as follows:

For each overlapping block in an image

_For each overlapping block in an image, generate and reconstruct a ridge orientation image by computing gradients of the pixels in the block, a ridge frequency image through obtaining the FFT value of the block, and an energy image by summing the power of FFT value.

_Apply the corresponding complex filter $h=(x+i y)^{m} g(x, y)$ centered at the pixel orientation in the orientation image, where ${ }^{g(x, y)}=e^{-\frac{x^{2}+y^{2}}{2 \sigma^{2}}}$ indicate the order of the complex filter and a Gaussian window, respectively.

_ Reconstruct the filtered image by composing filtered blocks

._The maximum response of the complex filter in the filtered

image can be considered as the reference point. Since there is only one output, the unique output point is taken as the reference point. The singular point detection algorithm in [30 is described as follows:

_Determine the core-delta path by determining the high angular variation points in the vertical direction. This mapping 
makes it possible to highlight the points with an angular variation closed to $\mathrm{p} / 2$ in the directional map.

_For each kernel (i, j), the differences computed among each

directional map element ( $i, j$ ), and its 8_neighbors are used to detect the zones with the highest vertical differences.

_Finally, the point having the maximum angular difference is selected.

\section{C) .Minutiae extraction}

Minutiae are extracted from the thinned fingerprint image.

A simple image scan generates a crossing number for each pixel. The crossing number $\mathrm{c} n(\mathrm{p})$ of a pixel $p$ in a binary image is defined as half the sum of the differences between pairs of the adjacent pixels in the 8-neighborhood of p:

$$
c n(p)=\sum_{i=1}^{8} P_{i \bmod 8}-P_{i-1}
$$

where $\mathrm{Pi}$ is a the binary value of the pixel (i) ( 0 for background and 1 for a ridge pixel) and $\mathrm{P} 0, \mathrm{P} 1, \ldots, \mathrm{P} 7$ are the

pixels belonging to an ordered sequence of pixels defining the eight neighborhood of $\mathrm{p}$. Intermediate ridge point if $\mathrm{c} n(\mathrm{p})=2$, a ridge ending minutia if $\mathrm{c} n(\mathrm{p})=1$, a bifurcation minutia if $\mathrm{cn}(\mathrm{p})=3$, or a more complex minutia (e.g., crossover) if $\mathrm{c} \mathrm{n}(\mathrm{p})>3$.

\section{D) Fingerprint features representation}

The features of the fingerprint will be represented by the number of minutiae of each type within a specific distance from the core point. This is achieved by dividing the fingerprint image into concentric tracks around the core point. The track width is chosen to be 10 pixels as in a 500 DPI image resolution, a ridge is around 5 pixels wide and the inter-ridge distance is also around 5 pixels. A vector of $\mathrm{N} * 2$, where $\mathrm{N}$ is the maximum number of tracks, can be constructed around the core point and can be obtained as follows:

$$
N=\left\lfloor\frac{\min \left(x c, y_{c}, W-x_{c}, H-y_{c}\right)}{10}\right\rfloor
$$

where $\mathrm{xc}$ and $\mathrm{yc}$ are the coordinates of the core point from the top left corner of the image, $\mathrm{W}$ is the width of the fingerprint image and $\mathrm{H}$ is its height.

\section{E)Vector distance matching algorithm}

The proposed matching algorithm is based on calculating the distance between the feature vector of the fingerprint (called the challenge fingerprint) and a set of pre-stored templates in the FIS database. This can be done in 2 scenarios,

1. In a one to one matching, a fingerprint feature vector is Compared to the set of pre-stored template(s) vector(s) of a specific person where the person ID is given as an input toa the matching system. If the (average) distance falls below a certain threshold, the finger print is matched

2. In a one to many matching, a fingerprint feature vector is compared to the set of the pre-stored templates vectors. The minimum (average) distance between the fingerprint image and the set of templates for a specific user is identified. If this (average) distance falls below a certain threshold ,the finger print is matched to the owner of the template set, otherwise no match is achieved.

\section{Conclusion}

In this paper three methods for low quality finger print enhancement are introduced. "Unsharp masking using quadratic filter for the enhancement of finger prints in noisy background" This method summarizes the design and implementation of a quadratic edge detection filter based on Volterra series. "TwoStage Enhancement Scheme for Low-Quality fingerprint Images by Learning From the Images" in this method 
to remedy the ridge areas and enhance the contrast of the local ridges, " a medium resolution fingerprint matching system" novel minutiae based fingerprint matching system is proposed.

\section{References}

[1]. M. D. Marsico, M. Nappi, and G. Tortora, "NABS: Novel approaches for biometric systems," IEEE Trans. Syst.,Man, Cybern. , Appl. Rev., vol. 41, no. 4, pp. 481-493, Jul. 2011.

[2]. V. Conti, C. Militello, F. Sorbello, and S. Vitabile, "A frequency-based approach for features fusion in fingerprint and iris multimodal biometric identification systems," IEEE Trans. Syst., Man, Cybern. C, Appl. Rev.,vol. 40, no. 4, pp. 384-395, Jul. 2010.

[3]. A. K. Jain, A. Ross, and S. Pankanti, "Biometrics: A tool for information security," IEEE Trans. Inf. Forensics Security, vol. 1, no. 2, pp. 125-143,Jun. 2006

[4]. S.K. Mitra, G. Sicuranza, Nonlinear Image Processing, Academic Press Series in Communication, Networking and Multimedia, San Diego, 2001.

[5]. R.Fletcher,M.J.D.Powell,Arapidlyconvergentdescentmethodforminimiza-tion, ComputerJournal6(1963)163-168

[6]. F.R.Gantmacher, TheTheoryof Matrices, Chelsea,NewYork

[7]. L. Hong, Y. Wang, and A. K. Jain, "Fingerprint image enhancement: Algorithm and performance evaluation," IEEE Trans. Pattern Anal.Mach.Intell., vol. 21, no. 4, pp. 777-789, Aug. 1998.

[8]. R. C. Gonzalez and R E.Woods, Digital Image Processing. Englewood Cliffs, NJ: Prentice-Hall, 2004.

[9]. P.Alper,A consideration of the discrete Volterra series, IEEE Transactions on Automatic Control AC-8(1963)322-327.

[10]. S.Y.Fakhouri, Identification of the Volterra kernels of nonlinear systems, IEEE Proceedings 127(6)(1980)296-304.

[11]. Conti V, Militello C, Sorbello F, Vitabile S. A frequency-based approach for features fusion in fingerprint and iris multimodal biometric identification systems. IEEE Trans Syst, Man, Cybernet2010;40(4):384-95. 